\title{
Parameters Governing Regolith Site Work by Small Robots
}

\author{
K. Skonieczny ${ }^{1}$, D. S. Wettergreen ${ }^{1}$ and W. L. "Red" Whittaker ${ }^{1,2}$ \\ ${ }^{1}$ Field Robotics Center, Robotics Institute, Carnegie Mellon University, 5000 \\ Forbes Avenue, Pittsburgh, PA 15213; email: kskoniec@cmu.edu \\ ${ }^{2}$ Astrobotic Technology Inc., 4551 Forbes Avenue, Suite 300, Pittsburgh, PA \\ 15213
}

\begin{abstract}
Continued exploration of the Moon and Mars will call for mobile robots that are increasingly capable in regolith; not only traversing it without getting stuck, but also manipulating and shaping it to suit mission needs. This work distinguishes issues governing performance of regolith operations, based on tasks motivated by preparatory site work for lunar outposts. Payload ratio (pound-forpound regolith-moving capacity) of small site work robots is identified as the key parameter governing metrics such as completion time for tasks ranging from berm building to trench digging. In addition to payload ratio, driving speed also governs berm building with small robots, while soil interaction parameters including regolith cohesion and soil-tool friction dictate trench digging performance. Furthermore, 3-dimensional bucket modeling highlights cohesion and friction as risks to the viability of using the smallest proposed class of excavation robots (those with mass of $250 \mathrm{~kg}$ or less). For trench digging especially, machines with higher mass (500 kg or more) may be required. Results are based on parameter sensitivity analyses using REMOTE, a task-level site work simulator.
\end{abstract}

\section{INTRODUCTION}

Effective interaction with regolith is an important ability for exploring other worlds. Whether blazing trails in search of scientific samples, building thermal shelters, or preparing berms and roads for an eventual human outpost, the productivity of regolith-moving machines will determine mission success.

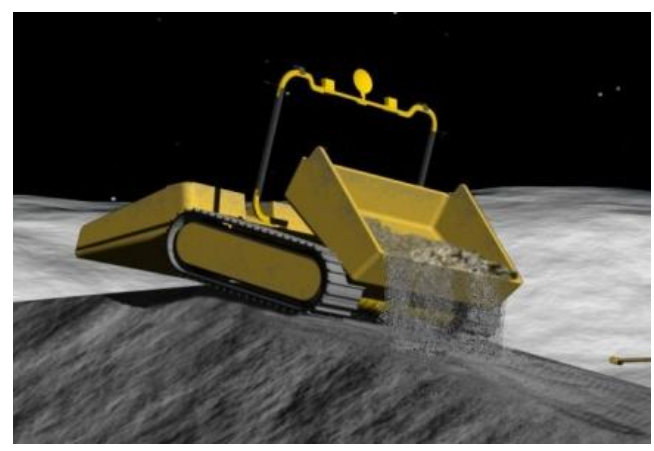

Figure 1. A conceptual robot building a berm on the Moon 
The scope of this study includes berm building and trench excavation, representative site work tasks that span a range of regolith interactions. Building a berm involves shallow digging, substantial material transport, and dumping onto a pile. During exploration, a berm-like structure could insulate a boulder with high heat capacity to transform it into a thermal shelter. In the context of lunar outposts, berms could be used to shield assets from regolith sandblasting caused by nearby rocket landings and takeoffs. Trench excavation involves deeper digging, and dumping of material nearby. Trenches at lunar outposts could be required to bury cables, power stations, or habitats.

This study specifically investigates the feasibility of performing site work using robots of mass up to $500 \mathrm{~kg}$. This work takes the liberty of defining such robots as small excavation robots, recognizing that "small" may be understood differently in other contexts. To keep comparisons between robots of different mass fair, total mass available is kept constant (for example, tasks are completed with one $500 \mathrm{~kg}$ robot or with two $250 \mathrm{~kg}$ robots).

Past trade studies have examined the applicability of various excavation robot options to lunar outpost site work. Boles et al [Boles 1993] compared the probable required launch mass of several construction machine suites. They concluded that typical terrestrial excavation machines would not be as effective as tripod cranes, sweeper leveller/excavators, and other innovative vehicles. Abu El Samid's work [Abe El Samid 2009] continued along the lines of Boles', concentrating on tradeoffs between autonomous and tele-operated operation and between single vehicle and team configurations. The author recommended a team of autonomous bulldozers for the task of berm building. Mueller and King's study [Mueller 2008] scored excavator designs on a number of quantitative and qualitative metrics and decided a multi-purpose machine with blade and excavator arm was most appropriate for lunar site work. Other researchers have reasoned in favor of pneumatic regolith extraction machines [Zacny 2007] or scraper/loader vehicles [Caruso 2006].

Each of the studies discussed above arrives at different conclusions regarding designs of lunar site work robots. Such varying results highlight the effect that differing assumptions, models, and metrics can play in such a complex problem. This study simulates lunar regolith-moving tasks. Parameters that govern site work performance are identified through sensitivity analysis, with particular attention paid to results that hold across model and metric selections.

\section{TASK-LEVEL SITE WORK SIMULATION}

Regolith-moving machines are commonly characterized for elemental actions like digging or driving [Wilkinson 2007] but what matters is the performance of a machine that combines digging and driving to achieve a comprehensive task. A task model is developed for regolith-moving tasks, such as berm building or trench excavation, that includes digging, transporting, dumping, and shuttling for recharge.

The task simulator, REMOTE (Regolith Excavation, MObility \& Tooling Environment), computes metrics including task completion time, production ratio, and production efficiency, based on parameters describing the task, the robotic system, and the environment. The novelty of comprehensive task simulation is that it identifies parameters that are important for overall task success. This determines which parameters matter most for system design and tradeoffs. 


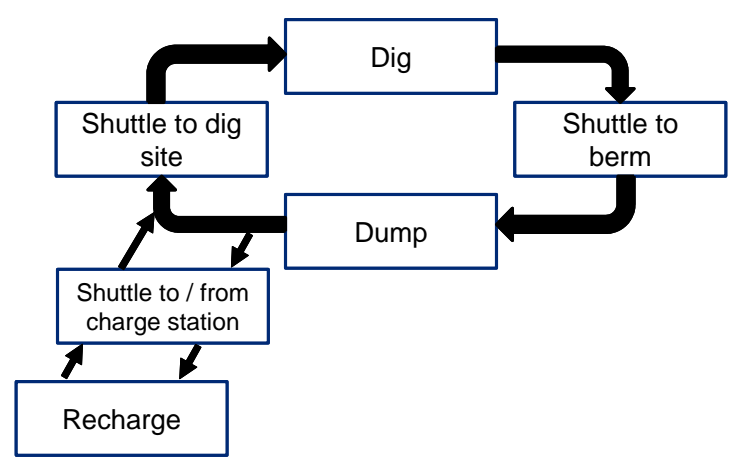

Figure 2. Task model for lunar regolith site work

Traction and excavation forces are modeled to determine admissible bucket geometries, and transport and recharge times are estimated based on driving speed and power draw. The underlying traction model is that of Bekker and Wong [Wong 2001], based on their theoretical and empirical work.

REMOTE offers a choice of underlying excavation models, which relate digging forces to bucket and soil parameters. One of the options is Luth-Wismer (Viking model) [Luth 1971, Wismer 1972], which was developed empirically from separate experiments in cohesive clay and cohesionless sand. The Viking model represents an excavating bucket by a single plate, and does not explicitly include soil friction angle or external (soil-tool) friction. The other excavation model option in REMOTE is Balovnev's [Balovnev 1983], which is a 3-D bucket model developed from theory. External friction contributes resistance on the bucket sidewalls. Model options allow for comparing results depending on inherent assumptions

Additional details of the implementation of REMOTE have been presented in past work [Skonieczny 2009]. Experimental validation and calibration is required before results should be used for explicit prediction of robot performance. Of more immediate utility is REMOTE's sensitivity analysis capability. Here it is not so much the values themselves of the calculated metrics that are paramount, but rather how sensitive these calculations are to changes in system, concept of operations, and environmental parameters.

\section{PARAMETRIC SENSITIVITY ANALYSIS}

This work analyzes the sensitivity of task completion time, production ratio (weight of regolith moved per hour, normalized by robot weight), and production efficiency (weight of regolith moved per unit of energy spent, normalized by robot weight) to the parameters in REMOTE. Parameters for sensitivity analysis include system parameters (such as individual robot mass, payload ratio - ratio of weight of regolith carried to vehicle weight, wheel radius, etc.) and concept of operations parameters (operational efficiency, distance to recharge station, etc.) that could be variables in system/mission design. The sensitivity analysis also includes regolith parameters (bulk density, cohesion, etc.) whose values are known within bounds. Each parameter is varied individually from its expected baseline value to maximum and minimum values in turn. The resulting values of the metrics are noted for each variation.

Figure 3 shows sensitivity of production ratio to relevant parameters for a berm building task. The task involves shallow digging, to a total depth of $30 \mathrm{~cm}$, 
over a large area (50 m diameter circle). Excavated material is moved to an arc along the circle and dumped in a berm. Average distance between dig and dump is $25 \mathrm{~m}$. The results shown in the figure are for an analysis using the Viking (Luth-Wismer) excavation model.

With all parameters set to their baseline expected values, the resulting production ratio is approximately $0.0025 \mathrm{hr}^{-1}$. For each parameter ( $\mathrm{Y}$ axis in Figure 3 ), changes in value can either increase or decrease production ratio ( $\mathrm{X}$ axis). The resulting values of the metric are shown by light bars (decrease in value from baseline) and dark bars (increase in value over baseline). The parameter values that result in these values are shown next to the bars. For some of the key sensitivity parameters, the baseline value is also listed (boxed values). For example, the baseline value of driving speed is $20 \mathrm{~cm} / \mathrm{s}$. Increasing speed to $50 \mathrm{~cm} / \mathrm{s}$ results in an increase in production ratio to more than $0.005 \mathrm{hr}^{-1}$, while decreasing it to $5 \mathrm{~cm} / \mathrm{s}$ causes a decrease in production ratio to below $0.001 \mathrm{hr}^{-1}$.

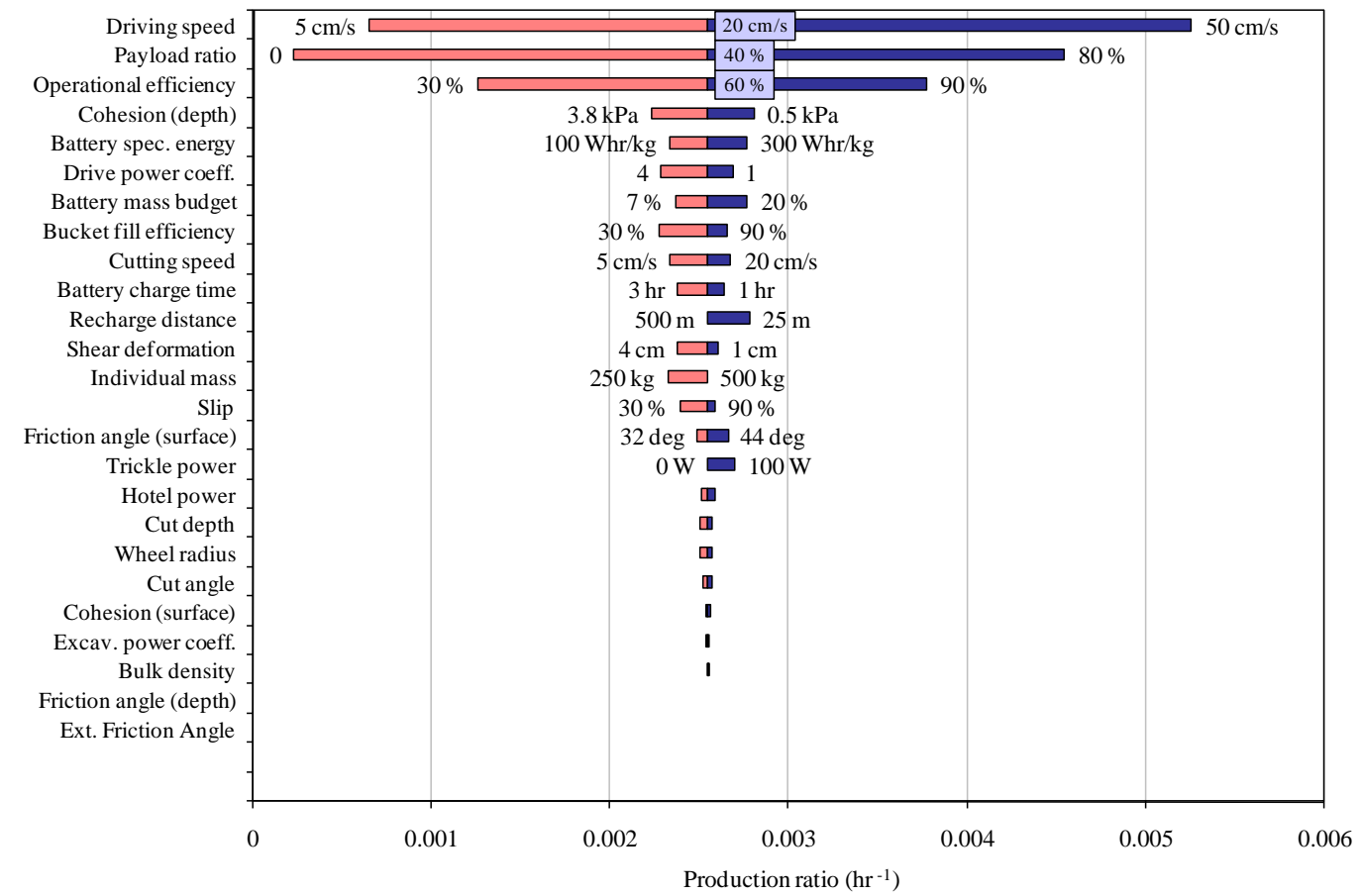

Figure 3. Parametric sensitivity for berm building (Viking model)

It is clear from Figure 3 that production ratio for berm building is significantly more sensitive to driving speed, payload ratio, and operational efficiency, than to other parameters. There are numerous parameters to which production ratio for berm building exhibits very little sensitivity, such as regolith bulk density, cut angle, and wheel radius.

Trench excavation is a considerably different site work task from berm building. It involves deeper digging, and the excavated material does not need to be transported a great distance. To analyze the sensitivity of key metrics to relevant parameters for trench excavation, a total digging depth of $60 \mathrm{~cm}$ and a transport distance of $2 \mathrm{~m}$ is used. Figure 4 and Figure 5 show results of this sensitivity analysis using the Viking and Balovnev excavation models, respectively. 


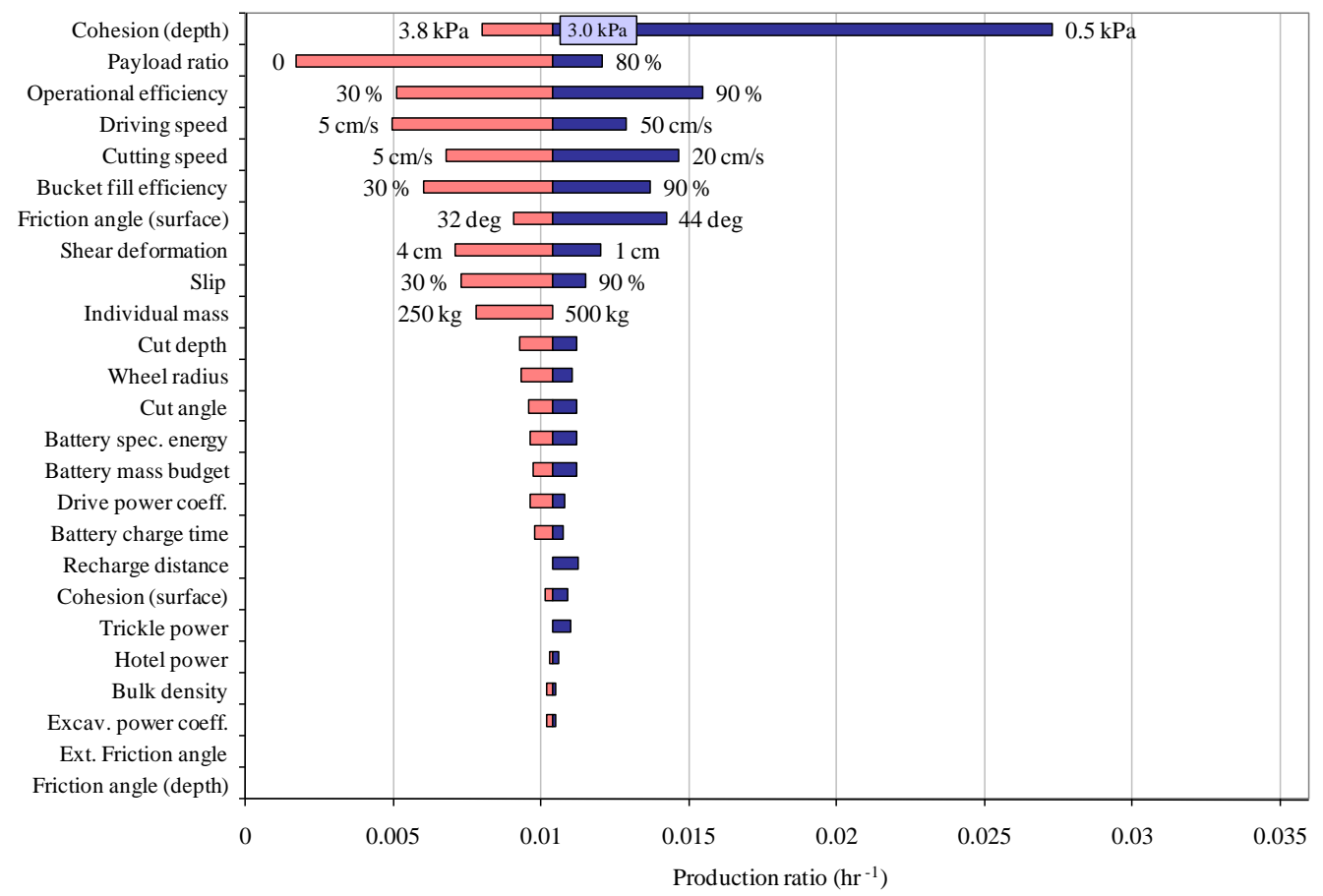

Figure 4. Parametric Sensitivity for Trench Excavation (Viking model)

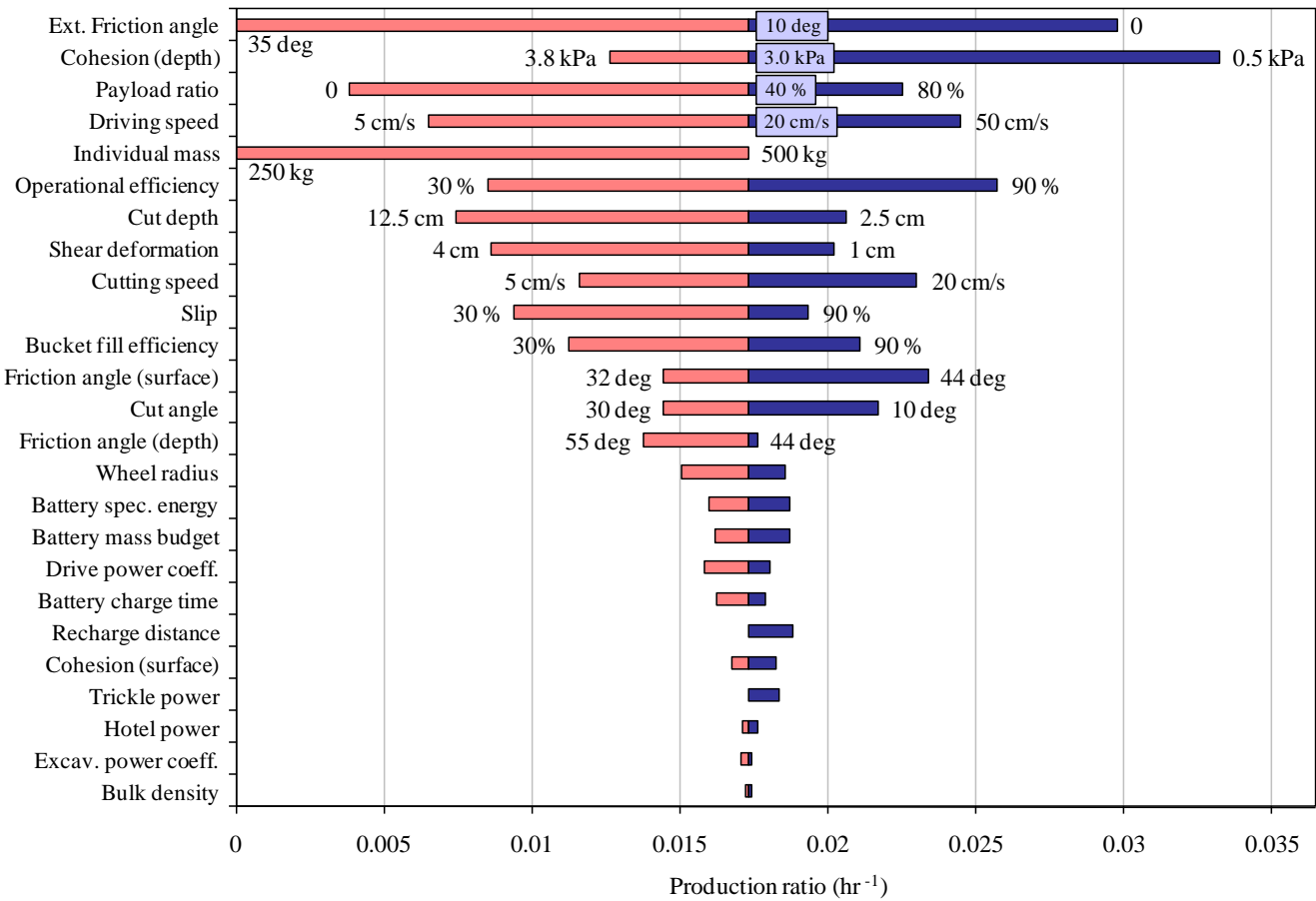

Figure 5. Parametric Sensitivity for Trench Excavation (Balovnev model)

Comparing Figure 4 to Figure 5 illuminates similarities and differences between assumptions of the Viking and Balovnev excavation models. The parameters exhibiting highest sensitivity generally correspond well across models. In the trench excavation sensitivity analysis, for example, regolith cohesion and payload ratio figure prominantly in the results regardless of the model used. On the other hand, the Balovnev model exhibits great sensitivity to the external friction angle (i.e. soil-tool friction), while the Viking model does not even 
include this parameter (resulting in zero sensitivity, by definition). Another difference is that the Balovnev model generally exhibits higher sensitivity to all parameters, as witnessed by the wider spread of production ratio values in Figure 5 compared to Figure 4.

Overall, the choice of excavation model has less effect on sensitivity analysis results than the choice of site work task. Table 1 and Table 2 show the parameters exhibiting highest sensitivity for berm building and trench excavation, respectively. The parameters are denoted in the table as follows: Payload ratio $(\mathrm{PR})$, driving speed $\left(\mathrm{v}_{\mathrm{d}}\right)$, operational efficiency $\left(\eta_{\mathrm{op}}\right)$, driving power coefficient $\left(\mathrm{K}_{\mathrm{Pd}}\right)$, external friction angle $(\delta)$, cohesion $(\mathrm{c})$, and individual robot mass $(\mathrm{m})$.

Table 1. Highest sensitivity parameters for berm building task.

\begin{tabular}{lccc}
\hline \hline Excavation model & Completion time & Production ratio & Prod. efficiency \\
\hline Viking & $\mathrm{PR}, \mathrm{v}_{\mathrm{d}}, \eta_{\mathrm{op}}$ & $\mathrm{v}_{\mathrm{d}}, \mathrm{PR}, \eta_{\mathrm{op}}$ & $\mathrm{PR}, \mathrm{K}_{\mathrm{Pd}}, \mathrm{v}_{\mathrm{d}}$ \\
Balovnev & $\delta, \mathrm{PR}, \mathrm{v}_{\mathrm{d}}, \eta_{\mathrm{op}}$ & $\mathrm{v}_{\mathrm{d}}, \mathrm{PR}, \delta, \eta_{\mathrm{op}}$ & $\mathrm{PR}, \delta, \mathrm{K}_{\mathrm{Pd}}, \mathrm{v}_{\mathrm{d}}$ \\
\hline \hline
\end{tabular}

Table 2. Highest sensitivity parameters for trench excavation task.

\begin{tabular}{lccc}
\hline \hline & Completion time & Production ratio & Prod. efficiency \\
Excavation model & & & \\
\hline Viking & $\mathrm{PR}, \eta_{\mathrm{op}}, \mathrm{v}_{\mathrm{d}}$ & $\mathrm{c}, \mathrm{PR}, \eta_{\mathrm{op}}$ & $\mathrm{c}, \mathrm{PR}, \mathrm{K}_{\mathrm{Pd}}$ \\
Balovnev & $\delta, \mathrm{m}, \mathrm{PR}$ & $\delta, \mathrm{c}, \mathrm{PR}$ & $\delta, \mathrm{PR}, \mathrm{c}, \mathrm{m}$ \\
\hline \hline
\end{tabular}

The most significant difference between results using the two excavation models is the importance of external friction in the Balovnev model. Additionally, individual robot mass surfaces as a high sensitivity parameter for trench digging when using the Balovnev model. Task simulation of trench digging using a smaller class of machines (two $250 \mathrm{~kg}$ robots), in fact, does not result in a feasible solution. The Balovnev model thus highlights a risk associated with using such a class of machines, though this risk is not confirmed by the Viking model.

It is possible to overestimate sensitivity to a given parameter by choosing an arbitrarily large range of values to vary over. Parameter value ranges were selected judiciously based on applicable space missions and analogue systems. A description of the selection process for two particularly high sensitivity parameters, payload ratio and driving speed, is given in the pursuant subsections.

Payload Ratio. Payload ratio is a parameter that can be designed for a vehicle equipped with a dump bed, by altering the size of the bed. The expected value used in REMOTE is $30 \%$, meaning the dump bed is expected to store and transport $30 \%$ of the mass of the robot in regolith. The expected value is suggested by the carrying capacity of a small terrestrial analogue robot, Cratos, which has a mass of $80 \mathrm{~kg}$ and carries a load of $23 \mathrm{~kg}$ [Caruso 2006].

The range of values studied for payload ratio spans from $0 \%$ to $80 \%$. $0 \%$ payload ratio simply means that no dump bed is present, and that only the excavation bucket is available for regolith transport. The upper bound of $80 \%$ for payload ratio is based on terrestrial construction examples. Skid steer, wheel, and track loaders attain payload ratios up to $80 \%$ [Caterpillar 2002]. Space systems, 
though, are subject to additional constraints that make it challenging to attain payload values that are as high as this upper bound.

Driving speed. The range of values studied for driving speed spans from $5 \mathrm{~cm} / \mathrm{s}$ to $50 \mathrm{~cm} / \mathrm{s}$. The lower bound is based on the top speed currently attainable by the Mars Exploration Rovers. An improvement in rover velocity over the current Mars rovers is expected when operating on the Moon, as solar flux is tripled and communication bandwidth and delay are appreciably improved. The upper bound of driving speed is based on performance of the teleoperated Lunokhod rovers [Kassel 1971]. Lunokhod 2 operated frequently at speeds on the order of $50 \mathrm{~cm} / \mathrm{s}$. These missions demonstrate the feasibility of teleoperating lunar rovers at such speeds, though construction sites introduce additional hazards and complexity that were not encountered during these missions.

\section{CONCLUSIONS}

Payload ratio. The ratio of weight of regolith carried to robot weight (a measure of pound-for-pound regolith-moving capacity) governs metrics ranging from task completion time to production ratio and production efficiency (weight of regolith moved per hour and per unit of energy spent, respectively, normalized by vehicle weight) for small site work robots. All these metrics exhibit strong sensitivity to payload ratio during both berm building and trench excavation, regardless of the excavation model assumed.

Driving speed. During berm building tasks, approximately $3 / 4$ of the operating time is devoted to transporting regolith. For long-haul tasks involving so much driving, a site work vehicle's speed has strong influence on overall performance. All key metrics exhibit robust sensitivity to driving speed, regardless of excavation model assumed, in the particular context of berm building.

Soil interaction parameters. Soil parameters such as cohesion and soil-tool friction greatly influence regolith production ratio and production efficiency during trench excavation. Both the Viking and Balovnev excavation models reveal cohesion as a significant parameter in trench digging performance. Soiltool (i.e. external) friction is not included in the Viking model, but is prominent in sensitivity results using Balovnev.

Viability of small robots for site work. Task-level simulation leads to mixed results concerning the viability of using the smallest class of robots (those with mass of $250 \mathrm{~kg}$ or less) for lunar regolith site work, depending on the choice of underlying excavation model. Sensitivity analyses incorporating the Viking model do not present any concerns, but those using the Balovnev model only lead to feasible solutions when regolith cohesion, soil-tool friction, or bucket cut depth are set to the low end of their expected value range. For larger vehicles, over 500 $\mathrm{kg}$, these risk factors are mitigated. The inconclusive nature of these particular results call for empirical investigation of the effects on excavation resistance of varying cohesion and soil-tool friction over a range of values relevant to a lunar context. 
Small robot designs for site work. Task-level sensitivity analysis illuminates parameters that merit particular attention when designing small robots for lunar site work. Lightweight dump beds for transporting regolith are features suggested by the importance of payload ratio. A transmission for switching between high power for digging and high speed for driving would be advantageous for vehicles performing tasks involving long hauls, like berm building.

Future work. The next step in this work is to measure the sensitivity of the identified governing parameters experimentally. Field tests with a representative site work robot will vary not only payload ratio and driving speed, but also parameters not thought to contribute great sensitivity, such as bucket cut angle. Thus sensitivity analysis will be calibrated to empirical results. Experiments directly investigating the impact of soil-tool friction on excavation performance are also recommended. Finally, a framework for designing and evaluating lunar site work robots would be of great benefit to the research community. An agreed set of metrics and assumptions could provide a common starting point from which questions of suitable configurations for real lunar outposts can be addressed.

\section{REFERENCES}

Abu El Samid, N. (2009). "Infrastructure robotics: A trade-off study examining both autonomously and manually controlled approaches to lunar excavation and construction." MASc Thesis, University of Toronto.

Balovnev, J. I. (1983). New methods for calculating resistance to cutting of soil, Amerind (translated from Russian), New Delhi.

Boles, W. W., Ashley, D. B., and Tucker, R. L. (1993). "Lunar-base construction equipment and methods evaluation." J. Aerosp. Eng., 6(3), 217-235.

Caruso, J. J., Spina D. C., Greer, L. C. et al (2006). "Excavation on the moon: regolith collection for oxygen production and ourpost site preparation." $46^{\text {th }}$ AIAA Aerospace Sciences Meeting and Exhibit, Reno, NV.

Caterpillar Inc. (2002). Caterpillar performance handbook, $32^{\text {nd }}$ edn., Caterpillar Inc., Peoria, IL.

Kassel, S. (1971). "Lunokhod-1 Soviet lunar surface vehicle." RAND Tech Report $R-802-A R P A$

Luth, H. J., and Wismer, R. D., (1971). "Performance of plane soil cutting blades in sand." Trans. ASAE, 14(2), 255-262

Mueller, R., P., and King, R. H., (2008). "Trade study of excavation tools and equipment for lunar outpost development and ISRU." AIP Conf. Proc., 969, 237-244.

Skonieczny, K., DiGioia, M. E., Barsa, R. L. et al (2009). "Configuring innovative regolith moving techniques for lunar outposts." IEEE Aerospace Conference, Big Sky, MT.

Wilkinson, A., and DeGennaro, A. (2007) "Digging and pushing lunar regolith: classical soil mechanics and the forces needed for excavation and traction." J Terramech., 44, 133-152.

Wismer, R., D., and Luth, H., J. (1972). "Performance of plane soil cutting blades in clay." Trans. ASAE, 15, 211-216.

Wong, J. Y., (2001) Theory of Ground Vehicles, $3^{\text {rd }}$ edn., Wiley, New York, NY.

Zacny, K., Mungas, G., Parrington, L., et al (2007). "Pneumatic drill and excavator for planetary exploration." $7^{\text {th }}$ Int. Conf. Mars, Pasadena, CA. 